\section{Referências bibliográficas}

1. Sanders JE, Buckner CD, Sullivan KM, Doney K, Applebaum F, Witherspoon R, et al. Growth and development in children after bone marrow transplantation. Horm Res 1988;30:92-7.

2. Sanders JE, Buckner CD, Sullivan KM, Doney K, Applebaum F, Witherspoon R, et al. The impact of marrow transplant preparative regimens on subsequent growth and development. Sem Hem 1991,28:244-9.

3. Sanders JE, Pritchard S, Mahoney P, Amos D, Buckner CD, Deeg HJ, et al. Growth and development following marrow transplantation for leukemia. Blood 1986;68:1129-35.
4. Thibaud E, Rodrigues-Macias K, Trivin C, Espérou H, Michon $\mathrm{J}$, Brauner R. Ovarian function after bone marrow transplantation during childhood. Bone Marrow Transpl 1998;21:287-90.

5. Mertens AC, Ramsay NKC, Kowis S, Naghia JP. Patterns of gonadal dysfunction following bone marrow transplantation. Bone Marrow Transpl 1988;22:345-50.

6. Sanders JE, Hawley J, Levy W, Gooley T, Buckner CD, Deeg JH, et al. Pregnancies following high dose cyclophosphamide with or without high dose busulfan or total-body irradiation and bone marrow transplantation. Blood 1996;87:3045-52.

\title{
Efeito a curto prazo da posição prona na oxigenação de crianças em ventilação mecânica
}

\author{
Short-term effect of prone positioning on oxygenation of pediatric patients \\ submitted to mechanical ventilation
}

\author{
Eduardo Juan Troster*
}

\begin{abstract}
A sinovações médicas, com uma certa freqüência, são intervenções terapêuticas mais complexas e caras do que a terapia convencional. Uma exceção foi o uso de corticosteróides no pré-natal para facilitar a maturidade pulmonar fetal; talvez a posição prona na insuficiência respiratória aguda possa ser outra exceção.

$\mathrm{Na}$ avaliação crítica de uma nova intervenção terapêutica, os estudos com um delineamento de um ensaio clínico randomizado são os mais adequados. O grupo controle deve ser igual ao grupo de estudo, exceto para o fator a ser estudado. Os pacientes devem ter as mesmas chances de participar em ambos os grupos, e uma forma de garantir é a alocação aleatória nos dois grupos (randomização).

\section{OFDA (Food and Drugs Administra-}

tion), para liberar uma droga para uso clínico, exige que existam pelo menos dois ensaios clínicos randomizados com amostras de um número suficiente de pacientes que permitam conclusões com significância estatística e repercussão clínica. Os desfechos avaliados devem ter impacto na Saúde, isto é, redução da mortalidade e/ou da morbidade. Os desfechos intermediários, geralmente parâmetros fisiológicos, são interessantes, porém podem não ter impacto na Saúde; por exemplo, melhorar a oxigenação sangüínea ou a complacência pulmonar. Numa revisão recente de 30 anos no tratamento de SDRA (síndrome de desconforto respiratório agudo) no Critical Care Medicine, em 2000,

\footnotetext{
* Coordenador do CTI-Pediátrico do Hospital Israelita Albert Einstein, coordenador do CTI-Pediátrico do Instituto da Criança do HC FMUSP, Doutor em Medicina pelo Departamento de Pediatria da FMUSP.
}

demonstrou-se que o uso do óxido nítrico não tinha impacto em morbidade e mortalidade, apesar de melhorar a oxigenação sangüínea.

Os trabalhos demonstrando melhora na oxigenação com a posição prona são, na sua grande maioria, séries de casos sem grupo controle. Isso prejudica conclusões sobre a aplicabilidade clínica para intervenções terapêuticas com impacto na Saúde dos pacientes.

No presente número do Jornal de Pediatria vale a pena ler o artigo de Bruno et al. sobre o efeito a curto prazo da posição prona na oxigenação de crianças em ventilação mecânica. O delineamento do estudo é de um ensaio clínico não randomizado (cada paciente é seu controle). Dos 18 pacientes estudados, apenas 5 apresentaram uma melhora na $\mathrm{PaO}_{2} / \mathrm{FiO}_{2}$ acima de 20. A população estudada consistia predominantemente de afecções pulmonares obstrutivas (12 crianças) que, em alguns trabalhos, mostra que talvez seja um grupo para melhorar a oxigenação com a posição prona.

Num trabalho de Mure et al., realizado no Instituto Karolinska, em Estocolmo, Suécia, em 1997, foram estudados 13 pacientes adultos com insuficiência respiratória aguda grave causada por trauma, sepse, aspiração e queimadura. Onze pacientes tinham uma hipoxia grave $\left(\mathrm{PaO}_{2} /\right.$ $\mathrm{FiO}_{2}<80 \mathrm{mmHg}$ ). Doze pacientes responderam com melhora na oxigenação, sendo que destes 9 sobreviveram ${ }^{1}$.

Em 1997 Broccard et al. avaliaram a influência da posição corpórea na extensão e distribuição da lesão pulmonar experimental num modelo canino com ácido oléico 
de síndrome de desconforto respiratório agudo, utilizando volumes correntes elevados e PEEP. A falha de aplicar PEEP suficiente ao tecido pulmonar, lesado pelo excesso de pressão nas vias aéreas, depleção de surfactantes ou aspiração ácida, acentua a lesão tecidual induzida pelo ventilador. Os animais em posição prona tiveram menor lesão do que os em posição supina ${ }^{2}$.

Um outro trabalho foi realizado no Hospital for Sick Children, em Toronto, Canadá, por Kornecki et al. e publicado no Chest em 2001. O objetivo deste trabalho foi comparar a posição prona com a posição supina quanto ao efeito na oxigenação em crianças com insuficiência respiratória aguda. A UTI Pediátrica do Hospital for Sick Children tem 36 leitos pediátricos. Foram estudadas 10 crianças com um índice de oxigenação de 22, isto é, pressão média das vias aéreas $x \mathrm{FiO}_{2} / \mathrm{PaO}_{2}$. Após um período de estabilização na posição supina, os dados de base eram colhidos, e os pacientes eram randomizados em dois grupos num estudo de crossover. O grupo 1, seqüência supina/prona e o grupo 2, seqüência prona/supina. Cada posição era mantida por 12 horas. A mecânica respiratória e a resposta aguda ao óxido nítrico inalatório eram avaliadas. Os resultados foram que o índice de oxigenação melhorou significativamente na posição prona em relação à posição supina no período estudado de 12 horas. A complacência estática do sistema respiratório e a resistência não foram afetadas de forma significativa na mudança de posição. O óxido nítrico inalatório não teve efeito na oxigenação em ambas as posições. A diurese aumentou na posição prona, resultando num balanço hídrico significativamente melhor ${ }^{3}$.

Em 1997, Numa et al. realizaram um trabalho para avaliar o efeito das posições prona e supina na Capacidade Residual Funcional (CRF), oxigenação e mecânica respiratória em lactentes e crianças em ventilação mecânica. O trabalho foi realizado em 30 crianças sob bloqueio neuromuscular com afecções que incluíam 10 pacientes com doenças restritivas de moderada/grave, 10 pacientes com doenças obstrutivas e 10 controle. Foram avaliados os seguintes parâmetros: gasometria arterial, capacidade residual funcional, complacência e resistência. Houve uma melhora (estatisticamente significativa, porém clinicamente irrelevante) na oxigenação nos pacientes com afecções obstrutivas. Além disso, houve uma redução relevante na resistência, na posição prona, em crianças com afecções obstrutivas. Não houve melhora na complacência nem na capacidade residual funcional ${ }^{4}$.

A maioria dos trabalhos levantados no MedLine seguem este padrão de série de casos ou crossover prejudicando uma conclusão definida para nossa prática diária. $\mathrm{O}$ trabalho de Bruno et al. tem o mérito de ser um dos primeiros no nosso meio a serem publicados em Pediatria. Serve também como motivação para a realização de mais estudos nessa área.

\section{Referências bibliográficas}

1. Mure M, Martling CR, Lindahl SGE. Dramatic effect on oxygenation in patients with severe acute lung insufficiency treated in the prone position. Crit Care Med 1997;25:1539-44.

2. Broccard AF, Shapiro RS, Schmitz L, Ravenscraft MD, Marini JJ. Influence of prone position on the extent and distribution of lung injury in a high tidal volume oleic acid model of acute respiratory distress syndrome. Crit Care Med 1997;25:16-27.

3. Kornecki A, Frndova H, Coates AL, Shemie SD. A randomized trial of prolonged prone positioning in children with acute respiratory failure. Chest 2001;119:211-8.

4. Numa AH, Hammer J, Newth CJ. Effect of prone and supine positions on functional residual capacity, oxygenation, and respiratory mechanics in ventilated infants and children. Am J Respir Crit Care Med1997;156:1185-9.

\title{
Exposição pré-natal à cocaína
}

\author{
Fetal cocaine exposure
}

\author{
Ana Guardiola*
}

\section{$\mathrm{O}$} efeitos sobre o feto e o recém-nascido $(\mathrm{RN})$ pelo uso de cocaína na gestante constituem um importante tópico de saúde pública, devido à incidência relativamente alta do uso dessa droga no mundo ${ }^{1}$.

A cocaína é a benzoimetilecgonina, um derivado das folhas das plantas do gênero eritroxilon, que atravessa a

\footnotetext{
* Professora Adjunta de Neurologia da FFFCMPA. Doutora/Livre-Docente em Neurologia Infantil.
}

barreira hematoencefálica, assim como a placentária, atingindo concentrações cerebrais até quatro vezes as plasmáticas $^{2}$. Tem sérios efeitos sobre o Sistema Nervoso Central (SNC) e Periférico do adulto e do feto, podendo causar alterações estruturais do SNC, cognitivas e comportamentais $^{3-6}$.

São bem conhecidos os efeitos teratogênicos causados pela cocaína no cérebro em desenvolvimento, afetando a formação e a anatomia, atuando sobre os neurotransmisso- 